\title{
Segurança do paciente e aspectos éticos: revisão de escopo
}

Carlise Rigon Dalla Nora ${ }^{1}$, José Roque Junges ${ }^{2}$

1. Universidade Federal do Rio Grande do Sul, Porto Alegre/RS, Brasil. 2. Universidade do Vale do Rio dos Sinos, São Leopoldo/RS, Brasil.

\section{Resumo}

O objetivo deste estudo foi identificar aspectos éticos relacionados à segurança do paciente em serviços de saúde. Para isso, realizou-se uma revisão de escopo nas bases SciELO, Lilacs, Ibecs, Medline, Bdenf, Cinahl e Google Acadêmico em setembro de 2019. Foram incluídos na amostra artigos originais, relatos de experiência, estudos teóricos e editoriais. A revisão abrangeu 32 estudos, publicados entre 2004 e 2019. Da análise do corpus resultaram sete categorias: fatores organizacionais e da equipe; comunicação com o paciente; comunicação de incidentes; beneficência e não maleficência; justiça; autonomia; e elementos potencializadores da ética na segurança do paciente. Acredita-se que os resultados desta revisão podem ser úteis para sensibilizar profissionais de saúde para aspectos éticos que envolvem a segurança do paciente.

Palavras-chave: Ética. Bioética. Segurança do paciente. Erros médicos. Revisão.

\section{Resumen}

\section{Seguridad del paciente y aspectos éticos: revisión de alcance}

El objetivo de este estudo es identificar aspectos éticos relacionados con la seguridad del paciente en los servicios de salud. Para ello, se realizó una revisión de alcance en las bases SciELO, Lilacs, Ibecs, Medline, Bdenf, Cinahl y Google Académico en septiembre del 2019. Se incluyeron en la muestra artículos originales, relatos de experiencia, estudios teóricos y editoriales. La revisión incluyó 32 estudios publicados entre el 2004 y el 2019. Del análisis del corpus resultaron siete categorías: factores organizacionales y del equipo; comunicación con el paciente; comunicação de incidentes; beneficência y no maleficencia; justicia; autonomía; y elementos potenciadores de la ética en la seguridad del paciente. Se considera que los resultados de esta revisión pueden resultar útiles para sensibilizar a los profesionales de la salud hacia los aspectos éticos que implican la seguridad del paciente.

Palabras clave: Ética. Bioética. Seguridad del paciente. Errores médicos. Revisión.

\section{Abstract}

\section{Patient safety and ethical aspects: scoping review}

The objective was to identify the ethical aspects present in the context of patient safety in health services. This was a scoping review on the following databases: Lilacs, Medline, Ibecs, BDENF, CINAHL, SciELO and Google Scholar in September 2019. Original research papers, experience report, theoretical studies, and editorials were included. The review comprised 32 studies published between 2004 and 2019. Seven categories emerged from the analysis: organizational and team factors; communication with patient; incident reporting; beneficence and non-maleficence; justice; autonomy and potential elements of ethics in patient safety. The results of this review may be useful to improve the sensitivity of healthcare professionals working in patient care about the ethical aspects that involve patient safety.

Keywords: Ethics. Bioethics. Patient safety. Medical Errors. Review. 
A temática da segurança do paciente é emergente em todo o mundo, devido à maior preocupação com o respeito pela dignidade do paciente, a qualidade dos cuidados e a estabilidade do sistema de saúde ${ }^{1}$. A magnitude do problema demonstrada pelo elevado número de incidentes relacionados à assistência, com potencial de danos transitórios e permanentes, bem como custos individuais e sociais - justifica os esforços empregados em pesquisas sobre $o$ assunto ${ }^{1}$.

Entre os incidentes e fatores que contribuem para a ocorrência de eventos adversos não intencionais durante a assistência, destacam-se falhas no tratamento medicamentoso, no diagnóstico e na organização dos serviços de saúde, registros clínicos incompletos, falta de capacitação do pessoal e comunicação inadequada entre profissionais e usuários ${ }^{2}$. Todos esses fatores, quando analisados sob uma perspectiva ética, exigem reflexões tanto no âmbito do compromisso pessoal quanto no organizacional.

Ainda há uma lacuna no que se refere aos aspectos éticos envolvidos na segurança do paciente. E a questão não é ética apenas pelo respeito a cada paciente individual; o problema é também clínico e sanitário, visto que impacta a qualidade dos serviços e a sustentabilidade do sistema de saúde. Nesse sentido, considerando que um dos princípios da ética é "primeiro de tudo, não causar dano" (princípio da não maleficência), a questão da segurança é inescusável ${ }^{3}$. Segurança e eficiência são requisitos de qualidade do cuidado ${ }^{4}$.

Os profissionais têm obrigação de proteger seus usuários de danos prestando assistência de qualidade. Cuidados seguros e de qualidade, no entanto, só serão garantidos se os profissionais tiverem consciência de questões éticas. Por isso aspectos éticos da segurança deveriam ser enraizados de forma sistemática nos serviços de saúde, possibilitando o desenvolvimento das melhores práticas. Nesse contexto, este estudo visa conectar a ética e a segurança do paciente a partir de uma revisão de escopo. Os resultados dessa revisão são discutidos com base no referencial teórico da ética da responsabilidade de Max Weber ${ }^{5}$ e Hans Jonas ${ }^{6}$.

Max Weber ${ }^{5}$ analisa a passagem de uma política entendida como ação liberal independente, definida por atividades individuais, para uma política compreendida como exercício dependente de estruturas organizacionais de governos e partidos, conformando uma burocracia estatal que determina a ação. Se no primeiro caso a ética remete às convicções subjetivas do político, no segundo ela é pautada pelos resultados das ações políticas. Não importam tanto as convicções do indivíduo, mas a responsabilidade pelas consequências coletivas de suas ações. Por isso Weber defende que a ética da responsabilidade é um modelo mais adequado para a ética das ações políticas nos tempos atuais, e o mesmo pode se dizer do exercício ético das ações em saúde e do cuidado em relação à segurança do paciente.

Hans Jonas, por sua vez, desenvolve uma ética da responsabilidade para tempos tecnológicos ${ }^{6}$. Ao propor um novo modelo, Jonas aponta três elementos a se considerar num momento em que a tecnologia fundamenta a conformação da sociedade. Segundo o autor, para definir como é necessário agir, é importante ter como referência as consequências negativas das ações, levando em conta mais os efeitos do que as causas. Dessa premissa decorre que o futuro se torna uma referência central para saber como agir eticamente - não mais tanto o passado e o presente, como até então. Por fim, o terceiro elemento para uma ética da responsabilidade é a necessidade de uma crítica contínua da utopia como definidora do futuro, tendo presente sempre os resultados negativos, realistas das ações, numa espécie de heurística do medo ${ }^{6}$. Jonas define "responsabilidade" como ação não recíproca que inclui confiança, e nesse sentido envolve uma relação desigual. Assim, humanos fragilizados, que têm seu bem-estar, interesse e destino sob os cuidados de outros, têm obrigações definidas para com seus "responsáveis", de quem são objeto de controle.

A partir desses referenciais teóricos, este estudo tem como objetivo identificar e discutir aspectos éticos da segurança do paciente presentes no contexto dos serviços de saúde.

\section{Método}

Este artigo traz resultados de revisão de escopo $^{7}$ sistematizada de acordo com Levac, Colquhoun e O'Brien ${ }^{8}$, que estabelecem as seguintes etapas: 1) identificação da questão de pesquisa, 2) identificação de estudos relevantes, 3) seleção dos estudos, 4) extração de dados e 5) separação, 
sumarização e relatório de resultados ${ }^{7,8}$. O sexto passo (consulta a especialistas), considerado opcional, não foi utilizado.

A delimitação de escopo seguiu a estratégia mnemônica População, Conceito e Contexto (PCC). Os profissionais da saúde foram a população; o conceito de interesse foram os aspectos éticos da segurança do paciente; e o contexto analisado foi o dos serviços de saúde. Assim, a questão de pesquisa pode ser definida como: quais são os aspectos éticos presentes no contexto da segurança do paciente nos serviços de saúde? Serviram de fontes de dados os indexadores Scientific Electronic Library Online (SciELO) e Biblioteca Virtual em Saúde (BVS), que inclui as bases Literatura LatinoAmericana e do Caribe em Ciências da Saúde (Lilacs), Índice Bibliográfico Espanhol de Ciências da Saúde (Ibecs), Medical Literature Analysis and Retrieval System Online (Medline), Base de Dados de Enfermagem (Bdenf) e Cumulative Index to Nursing and Allied Health Literature (Cinahl). A ferramenta Google Scholar e listas de referências de textos relevantes na também foram verificadas.

Selecionaram-se os seguintes descritores controlados, registrados em Medical Subject Headings (MeSH) ou nos Descritores em Ciências da Saúde (DeCS): ethics; bioethics; professional ethics; patient safety; e medical errors. Os termos também foram buscados em sua equivalência em espanhol e português. A estratégia de busca utilizada seguiu a definição de cada base de dados correspondente. Utilizou-se o operador booleano "and" com as seguintes combinações: "ethics and patient safety"; "ethics and patient safety and bioethics"; "professional ethics and patient safety"; e "ethics and medical errors". Essas estratégias de busca, adotadas também em sua equivalência em espanhol e português, foram executadas em setembro de 2019.

Foram incluídos artigos originais, estudos teóricos, relatos de experiência, estudo de caso e editoriais, publicados em português, espanhol ou inglês, que tratassem de segurança do paciente e ética. Não se definiu um limite temporal. Textos duplicados, revisões, teses, dissertações e matérias jornalísticas foram excluídos. Para sistematizar o processo de inclusão, optou-se pela metodologia PRISMA Extension for Scoping Reviews (PRISMA-ScR) ${ }^{9}$. Os estudos foram pré-selecionados a partir da leitura dos títulos e resumos, e chegou-se à amostra final depois de leitura na íntegra dos artigos pré-selecionados (Figura 1).

Figura 1. Fluxograma da seleção dos estudos de acordo com o método PRISMA Extension for Scoping Reviews

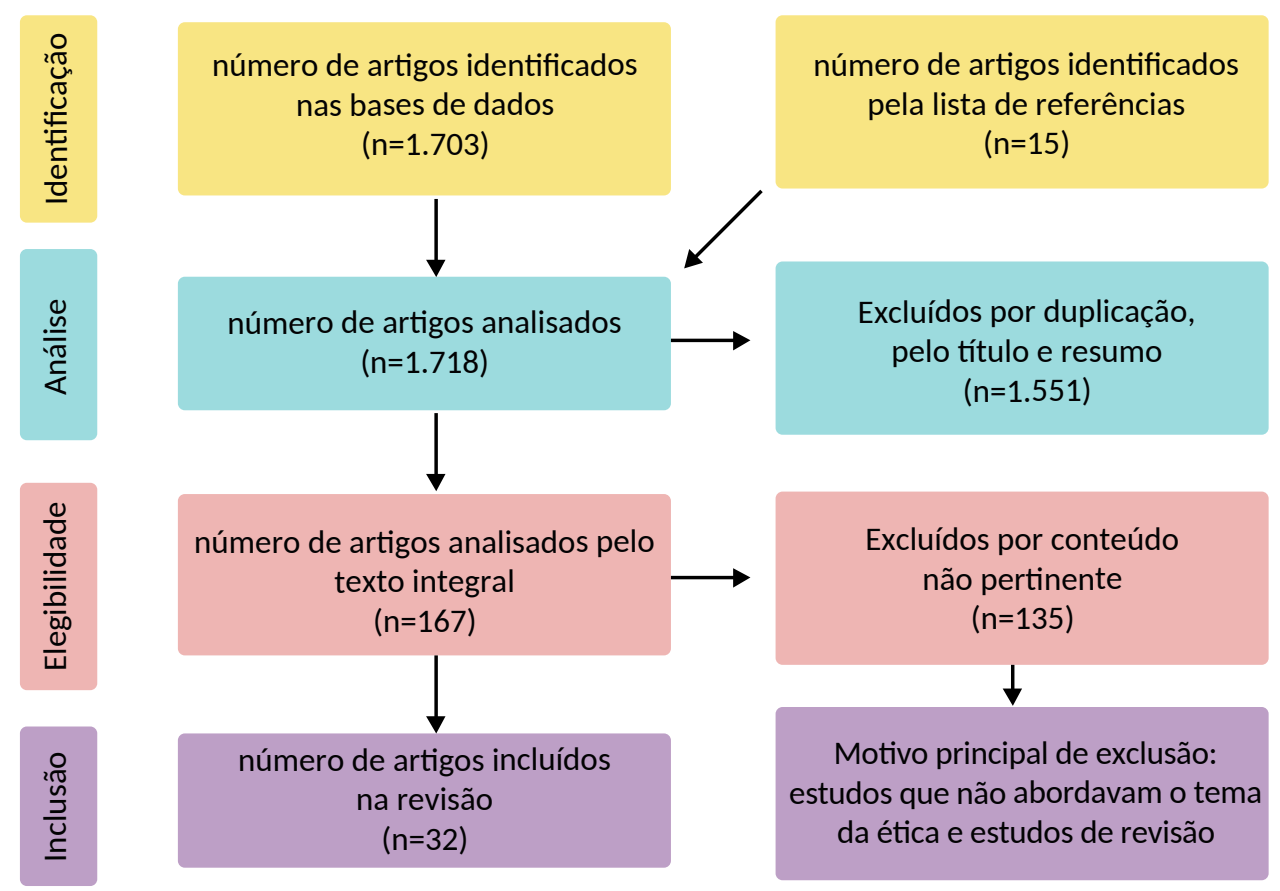


Para extrair os dados e identificar elementos essenciais dos estudos, utilizou-se instrumento estruturado no Microsoft Excel. Esse mapeamento permitiu sintetizar e interpretar os dados, gerando uma descrição numérica dos textos incluídos na revisão. Assim, procedeu-se à etapa de separação, sumarização e relatório de resultados com a intenção de apresentar uma visão geral de todo o material por meio de categorias temáticas.

\section{Resultados}

Após avaliação e seleção dos artigos, foram incluídos na revisão de escopo 32 estudos, publicados entre 2004 e 2019. Os resultados são apresentados a seguir, com uma descrição geral das características dos estudos, acompanhada de observações sobre sete categorias evidenciadas na análise do corpus: 1) fatores organizacionais e da equipe; 2) comunicação com o paciente; 3) comunicação de incidentes; 4) beneficência e não maleficência; 5) justiça; 6) autonomia; e 7) elementos potencializadores da ética na segurança do paciente.

\section{Descrição dos estudos}

A maioria dos estudos foi publicada no ano de $2015(n=4)^{10-13}$, seguido por $2019(n=3)^{14-16}$, $2017^{17,18}$, $2009^{19-21}$ e $2007^{22-24}$, com dois estudos cada. Os textos foram publicados em 28 periódicos científicos, dentre os quais apenas dois contaram com mais de uma publicação: Nursing Ethics $(n=4)^{16,25-27}$ e HEC Forum $(n=2)^{28,29}$. Quanto ao local, a maioria das pesquisas foi desenvolvida nos Estados Unidos ( $n=11)^{11,19,20,22,24,29-34}$, seguido por Brasil $(n=3)^{17,21,35}$, Espanha $(n=2)^{10,14,36}$ e Irã $(n=2)^{16,18}$. Outros países, como Austrália ${ }^{23}$, Canadá ${ }^{25}$, Colômbia ${ }^{37}$, Finlândiaa ${ }^{38}$, Índia ${ }^{12}$, Reino Unido ${ }^{15}$ e Suécia ${ }^{39}$ apresentaram apenas um texto. Dos 32 estudos, sete incluíram em sua amostra enfermeiros ${ }^{11,15-17,31,35,39}$, um incluiu gestores ${ }^{29} \mathrm{e}$ outro incluiu especialistas em bioética ${ }^{36}$.

\section{Fatores organizacionais e da equipe}

Essa categoria compreende fatores de organização dos serviços: carência de recursos, rotatividade, escassez de recursos humanos, falhas de gerenciamento, sobrecarga de trabalho e negligência do sistema de saúde. Já nos fatores relativos à equipe de profissionais, destacam-se: competência da equipe, déficit de conhecimento e desempenho prejudicado.

Os achados apontam que fatores organizacionais são os que mais afetam aspectos éticos da segurança do paciente. Tais fatores se relacionam com a incapacidade de oferecer o melhor atendimento por conta de carência de recursos ${ }^{1,32}$, alta rotatividade dos profissionais e escassez de recursos humanos no ambiente de cuidado ${ }^{13}$. Enfermeiros relataram que a maioria dos incidentes é causada por sobrecarga de trabalho ${ }^{15,27,32}$. No mesmo sentido, estudo que investigou incidentes de medicação identificou como principais causas a falta de atenção ou de conhecimento e a sobrecarga dos profissionais ${ }^{21}$.

Outro estudo aponta problemas de confidencialidade e privacidade do prontuário eletrônico relacionados ao uso de sistemas eletrônicos que fragmentam as informações, dificultando o acesso e tornando os dados mais imprecisos ${ }^{25}$. Já outra pesquisa ${ }^{5}$ refere que o profissional sobrecarregado tende a interpretar de maneira equivocada a prescrição médica registrada em prontuário clínico.

King ${ }^{31}$ demonstrou que a negligência sistêmica - incluindo fatores não médicos, como falta de vontade política e social - pode causar muitos danos a um sistema de saúde. Tal negligência é responsável por falhas como oferta irregular e baixa qualidade de medicamentos, diagnóstico incorreto, atraso no encaminhamento de pacientes para atendimento especializado, mau atendimento e falta de controle de infecções (caso, por exemplo, da disseminação da tuberculose em países africanos) ${ }^{31}$. Assim, eventos que deveriam ser considerados imperdoáveis em um sistema de saúde passam a ser aceitos como normais ${ }^{31}$.

Fatores pessoais ligados à atividade dos profissionais da saúde foram verificados em menor medida. Há menções a déficit de conhecimento e habilidades entre profissionais, a elementos que podem ameaçar a segurança do paciente ${ }^{6} \mathrm{e}$ à falta de competência da equipe ${ }^{1,32}$. Arksey e $0^{\prime}$ Malley $^{7}$ referem também risco de desempenho prejudicado devido a abuso de álcool e doença mental ou física, além de comportamentos abusivos ou destrutivos.

\section{Comunicação com paciente}

Nessa categoria destacam-se aspectos relacionados à comunicação com o paciente, com a família e 
com a equipe de saúde. Os estudos fazem referência tanto à comunicação adequada, com veracidade, clareza, transparência, sinceridade e honestidade, como à comunicação inadequada. Os resultados apontam que o principal benefício de uma boa comunicação é o aumento da confiança entre profissional, paciente e familiares ${ }^{36}$. Uma conduta destacada é o diálogo participativo, baseado em relações interpessoais simétricas ${ }^{40}$.

Kadivar e colaboradores ${ }^{18}$ destacam que é obrigação do profissional informar o paciente ou a família sobre cada evento indesejado, ou seja, o paciente lesado tem direito de saber o que aconteceu ${ }^{10,22,24,30}$. Giraldo e colaboradores ${ }^{36}$ concordam que se deve comunicar ao paciente não apenas o fato em si, mas também as causas e possíveis soluções para o erro. Nesse sentido, as características básicas de um pedido de desculpa deveriam ser sinceridade, clareza e honestidade. Cabe ao profissional revelar ao paciente o que aconteceu de maneira responsável, respeitando os princípios éticos da profissão e prezando o diálogo ${ }^{40}$. A resposta ética a um evento adverso começa pela humildade de revelar o erro honestamente ${ }^{13,24,34}$. Assim, permite-se que o paciente tome decisões informadas sobre futuras opções de tratamento ${ }^{22}$, ainda que a opção seja por interromper o tratamento na instituição onde ocorreu o erro ${ }^{34}$.

Pavlish e colaboradores ${ }^{11}$ assinalam que a falta de comunicação aberta com o paciente pode se dever a diferenças culturais, religiosas e morais. É comum também que a comunicação inadequada se deva à assimetria de conhecimentos, visto que, enquanto profissionais de saúde têm alto grau de especialização, pacientes e familiares muitas vezes enfrentam situações inéditas e podem ter dificuldade em entender as informações e tomar decisões relacionadas ao cuidado ${ }^{32}$. Nesse sentido, Milos e Larraín ${ }^{10}$ indicam que profissionais de enfermagem ocupam posição estratégica, uma vez que obtêm informações tanto do paciente como dos diversos profissionais da saúde.

\section{Comunicação de incidentes}

Essa categoria aborda questões relativas à comunicação do incidente ao paciente, à sua família e à instituição. Destacam-se os seguintes aspectos: medo de comunicar um evento adverso e receio de sofrer repressão, punição ou constrangimento.
O medo de consequências legais ou da reação da mídia e de colegas de profissão é uma das barreiras para a comunicação de incidentes ${ }^{36}$. Assim, é comum que profissionais não informem eventos adversos ao paciente e a outras instâncias com medo de repreensões, demissão ou perda de respeito pelos pares. Em alguns casos, os profissionais julgam os incidentes cometidos como de importância menor, como se não houvesse necessidade de relatá-los ${ }^{11}$.

Os resultados demonstram também que os profissionais sentem medo de sofrer processos por negligência ${ }^{30}$. Outro estudo, por sua vez, refere que profissionais evitam comunicar eventos adversos aos pacientes com a justificativa de que estes não estariam preparados para enfrentar a situação ${ }^{10}$. Coli, Anjos e Pereira ${ }^{35}$ chamam atenção ainda para o fato de que a formação acadêmica de enfermeiros e médicos reforça a premissa de um cuidado livre de incidentes, passando a mensagem de que erros são inaceitáveis. A cultura da punição torna impossível discutir os fatos de modo crítico e construtivo, de modo que mesmo profissionais que relatam erros honestamente continuam a ser tratados como culpados ${ }^{41}$.

Até mesmo profissionais em posição de liderança demonstram receio de se defender contra uma reivindicação legal, pois está é demorada, constrangedora e pessoalmente humilhante. Os achados, portanto, indicam que o medo é a principal barreira para a comunicação de eventos adversos ${ }^{34}$. A cultura do medo desestimula a divulgação de informações a um paciente que pode se tornar testemunha de acusação contra a instituição ou o profissional ${ }^{34}$.

\section{Beneficência e não maleficência}

Nessa categoria destacam-se aspectos relacionados aos princípios de beneficência (alcançar o bem) e não maleficência (minimizar danos causados ao paciente). A beneficência, portanto, refere-se à obrigação moral de prevenir danos ${ }^{24}$ e fazer o bem ${ }^{27}$, enquanto a não maleficência se refere à obrigação moral de não causar danos.

$A$ beneficência é colocada em xeque quando os incidentes não são relatados, pois isso impede que outros profissionais tenham acesso a essa informação e não cometam o mesmo erro ${ }^{24}$. Já o princípio da não maleficência é violado em todos os incidentes 
cometidos contra o paciente ${ }^{24,27}$. A falta de honestidade na comunicação e a falta de empenho em buscar soluções para incidentes adversos desrespeitam tanto a beneficência quanto a não maleficência ${ }^{33}$.

Respeitar o princípio da não maleficência envolve também aplicar orientações e procedimentos práticos para evitar danos (por exemplo: esterilizar materiais, lavar as mãos etc.) ${ }^{15}$. A falha em implementar essas práticas compromete a segurança do paciente, e o dever da não maleficência se aplica mesmo após o evento adverso ter ocorrido, porque a instituição de saúde pode causar danos ainda maiores ao não ser honesta com o paciente ${ }^{34}$. Por outro lado, respeitar o princípio da beneficência exige mais do profissional, que deve tomar medidas positivas para ajudar os outros, e não apenas se abster de atos prejudiciais. É preciso, por exemplo, manter-se atualizado e criar padrões de prática que promovam benefício ${ }^{15}$.

A ética e a segurança do paciente estão entrelaçadas. Espera-se que todos os pacientes sejam tratados com dignidade ${ }^{18,26}$ e se mantenham protegidos de qualquer dano possível ${ }^{18}$ - ou seja, é obrigação moral do profissional, em primeiro lugar, não fazer mal ${ }^{30}$. Assim, beneficência e não maleficência se conectam aos direitos do paciente, à segurança dos cuidados e a serviços de saúde que garantam atendimento livre de perigo ou risco de lesão ${ }^{42}$.

\section{Justiça}

Nessa categoria estão aspectos relacionados ao princípio da justiça: compromisso com o bem público, consciência de problemas e injustiças sociais, distribuição equitativa de bens e serviços e responsabilidade pela qualidade do cuidado.

Todo paciente tem direito a cuidados justos, equitativos e adequados. No entanto, desigualdades sociais, em particular no acesso a cuidados, tornam a discussão sobre justiça mais complicada. King ${ }^{31}$ define a injustiça como todo ato ou omissão que negue direitos ao paciente. Por outro lado, um exemplo de justiça mencionado pelo autor é a aplicação coerente dos padrões de prática de enfermagem para todos os pacientes, independentemente de situação econômica, educacional, cultural, religiosa, racial, idade ou diferenças sexuais ${ }^{31}$.

A distribuição equânime dos benefícios (o direito de o paciente de receber uma parte justa de benefícios e ônus e riscos) tem relação com o princípio de justiça ${ }^{34}$. Segundo tal princípio, o interesse do paciente precede a preocupação do profissional com consequências legais, de modo que não cabe à pessoa afetada arcar com o ônus da não comunicação de eventos adversos ${ }^{34}$.

Não fazer nada e permitir que pessoas inocentes sejam feridas e morram é uma injustiça não apenas para os imediatamente afetados, mas também para os pobres, impotentes e marginalizados que não têm acesso à assistência, uma vez que os custos com o sistema de saúde disparam por causa de incidentes adversos ${ }^{33}$. Assim, Clark $^{33}$ afirma que o desperdício de recursos em incidentes é uma grave injustiça contra todos os cidadãos. Por outro lado, minimizar os incidentes é dar segurança ao paciente e agir de modo justo ${ }^{33}$.

\section{Autonomia}

Nessa categoria, destacam-se aspectos relacionados à autonomia, ou seja, o que cada paciente ou família, no exercício de sua vontade, decide sobre seu próprio cuidado. Os resultados indicam que a autonomia se refere ao direito de escolha dos pacientes e à obrigação dos profissionais de respeitar essas escolhas ${ }^{31}$. Não se trata, no entanto, de um dever, mas de um direito do paciente ${ }^{31}$. Os exemplos incluem o consentimento informado e a recusa a tratamentos ${ }^{31}$. O tema da autonomia aparece em 7 dos 32 textos que formam o corpus da pesquisa $21,22,24,27,29,31,34$.

O paciente que pode compreender e raciocinar sobre riscos, benefícios e alternativas de um tratamento em particular tem capacidade suficiente para se engajar no processo de consentimento informado ${ }^{32}$. No entanto, o princípio da autonomia só é verdadeiramente respeitado quando o profissional cumpre o dever de garantir o direito do paciente de dirigir o curso da própria vida e tomar decisões sobre o próprio cuidado ${ }^{34}$. Nesse sentido, o respeito à autonomia é uma prestação de contas crucial para todos os profissionais, como se este fossem advogados do paciente ${ }^{24}$. Há duas responsabilidades simultâneas: equilibrar o dever de evitar riscos (segurança) com o respeito à autonomia (escolhas) ${ }^{27}$. Quando um incidente adverso não é comunicado, o paciente não tem as informações necessárias para se autodeterminar ${ }^{22}$. Consequentemente, sua liberdade para a tomada de decisão é cerceada. 
Nelson e colaboradores ${ }^{29}$ demonstraram que limitar direitos do paciente é a preocupação ética mais frequentemente identificada. Alguns exemplos de cerceamento da autonomia são: desrespeitar direitos de uma pessoa que tenha mostrado comportamento violento para com funcionários ou outros pacientes; restringir a disponibilidade de tratamento para usuários percebidos como "abusadores" do sistema; e diminuir opções de admissão ou de terapêuticas para pacientes que tenham história de má adesão ao tratamento ${ }^{29}$. Essas situações muitas vezes representam conflitos entre direitos pessoais (autonomia do paciente) e regras de segurança da instituição ${ }^{29}$.

\section{Elementos potencializadores da ética na segurança do paciente}

Essa categoria evidencia elementos que potencializam o papel da ética na segurança do paciente: educação ética, habilidade de comunicação, formação acadêmica, comitês, sistemas de notificação e cultura organizacional. Desses elementos, o que mais se destacou nas publicações foi a educação ética ${ }^{11,16,17,26,30}$. Kangasniemi e colaboradores ${ }^{26}$, por exemplo, propõem que o principal desafio da enfermagem é dar visibilidade a questões éticas relacionadas à segurança do paciente. Outro estudo, com líderes de equipes de enfermagem, sugere que instituições deveriam oferecer educação e elaborar políticas e práticas que promovam ações éticas e trabalho em equipe ${ }^{11}$.

A educação ética é também apresentada como grande desafio ${ }^{30}$. Barkhordari-Sharifabad e Mirjalili ${ }^{16}$ apontam que oferecer programas de treinamento para liderança ética em enfermagem, na forma de workshop, poderia ajudar a reduzir incidentes e manter a segurança do paciente. Outro estudo ${ }^{17}$ destaca a importância de formação continuada da equipe de enfermagem, com capacitação baseada em procedimentos operacionais e protocolos da área, para prevenir erros relacionados a medicação ${ }^{17}$.

Outro tema presente nos estudos é a necessidade de fomentar habilidades comunicativas ${ }^{29} \mathrm{e}$ o conhecimento jurídico dos profissionais antes da comunicação de incidentes, por meio de apoio e treinamento ${ }^{36}$. Assim, é fundamental implementar programas específicos de comunicação e de pedidos de desculpas que contem com suporte institucional ${ }^{43}$.

Outro elemento abordado pelos estudos analisados são os currículos da graduação, que deveriam contemplar conteúdos e competências necessárias para profissionais compreenderem a segurança do paciente, bem como deveres éticos e obrigações legais que integram a gestão do cuidado ${ }^{10,21}$. Arries ${ }^{25}$ também assinala a necessidade de mudanças curriculares para que enfermeiros desenvolvam competências essenciais, como habilidades em informática, prática baseada em evidências e melhoria da qualidade do cuidado.

$\mathrm{O}$ autor ainda propõe que profissionais e pacientes deveriam se envolver nos comitês de ética das instituições ${ }^{25}$. No mesmo sentido, Pavlish e colaboradores ${ }^{11}$ consideram que a confiança mútua entre equipe e gerência dos serviços é um elemento-chave para a qualidade e o caráter ético do cuidado.

Os achados indicam que incidentes adversos devem ser amplamente divulgados o mais rápido possível, para que os serviços possam alterar protocolos e evitar que acidentes semelhantes aconteçam no futuro ${ }^{30}$. Daí a necessidade de criar um sistema de notificação que respeite a confidencialidade dos profissionais que relatam $\operatorname{casos}^{24}$. O relatório de incidentes anônimo já é universalmente reconhecido como estratégia importante para melhorar as taxas de notificação de incidentes, reduzindo riscos e incidência de falhas nos serviços de saúde ${ }^{41}$.

Segundo Erlen ${ }^{22}$, as organizações precisam ser capazes de documentar e avaliar o que está realmente acontecendo a fim de identificar problemas e implementar novas práticas, ao invés de apenas culpar os indivíduos. As estratégias para reduzir incidentes incluem vigilância, mudança da cultura organizacional e criação de um ambiente seguro, isto é, um ambiente que disponha de relatórios que divulguem amplamente práticas inseguras e incidentes $^{22}$. Essas estratégias devem ser implementadas em todos os níveis, para todas as categorias profissionais, visto que nenhum profissional é imune a cometer falhass ${ }^{22}$. A divulgação de incidentes aos pacientes deveria ser parte da rotina de atendimento ${ }^{34}$. Por isso $\mathrm{Clark}^{33}$ sugere que legisladores criem políticas públicas para minimizar incidentes na saúde e proteger o paciente, priorizando a vida humana. 


\section{Discussão}

$\mathrm{Na}$ atualidade, a segurança do paciente é afetada por dois aspectos essenciais dos serviços de saúde: o organizacional, que estrutura as práticas do sistema, e o tecnológico, que determina o desempenho dessas práticas. As mudanças macropolíticas (organizacionais) e micropolíticas (tecnológicas) do trabalho têm profundas consequências no exercício ético das profissões, com implicações para a segurança do paciente. Essas transformações mudam radicalmente o foco da ética, que passa das intenções aos resultados. Isso representa a passagem de uma ética da convicção para uma ética da responsabilidade. O que interessa para o exercício ético da profissão é o resultado da ação para o paciente, dependente do aspecto organizacional e tecnológico do trabalho, e não tanto da intenção da ação. Assim, os profissionais são avaliados com base nos resultados para os destinatários das práticas. Por isso a responsabilidade torna-se categoria central.

A presente seção discute os dados apresentados com base na ética da responsabilidade de Max Weber ${ }^{5}$ e Hans Jonas ${ }^{6}$. As três primeiras categorias identificadas (fatores organizacionais e da equipe, comunicação com o paciente e comunicação dos incidentes) se relacionam com o aspecto organizacional das práticas de saúde e têm como referência ética a responsabilidade pelos resultados das ações, especialmente os resultados negativos para a segurança do paciente. Três outras categorias remetem a princípios da bioética (beneficência e não maleficência, justiça e autonomia) e se referem ao aspecto tecnológico das práticas de saúde. Esse aspecto demanda uma compreensão da responsabilidade como relação desigual, não recíproca, de respeito pela subjetividade do paciente como exigência para garantir sua segurança. Por fim, a última categoria (elementos potencializadores da ética na segurança do paciente) pode ser discutida criticamente quando se compreende a ética pela perspectiva da responsabilidade.

A categoria que se refere a fatores organizacionais tem relação com a competência da equipe, a falta de recursos, a rotatividade e a sobrecarga de pessoal. Nora, Zoboli e Vieira ${ }^{44}$ mostraram que trabalhar com colegas incompetentes pode aumentar a chance de vivenciar problemas éticos.
As consequências adversas não são causadas por intenção dos profissionais, mas resultam de fatores organizacionais que precisam ser avaliados eticamente, sob a perspectiva da responsabilidade do sistema, e não das convicções pessoais. Por isso tanto as falhas administrativas como a deficiência de conhecimento por parte dos profissionais são fatores organizacionais. Cabe destacar, ainda, que apesar de não dependerem das intenções ou convicções, os resultados adversos provocam sofrimento moral, uma vez que o profissional tende a ser responsabilizado por problemas de caráter organizacional.

$\mathrm{Na}$ categoria comunicação com o paciente, os itens mais frequentemente mencionados foram: clareza, transparência, honestidade e veracidade características que também dependem de fatores organizacionais, verificados e avaliados por resultados, e não por intenções. Tais características, portanto, devem ser vistas como referência ética para a responsabilidade dos gestores. Biasibetti e colaboradores ${ }^{45}$ corroboram essa afirmação ao mostrar que uma boa comunicação garante a qualidade e a segurança na assistência prestada. Já Nora, Zoboli e Vieira ${ }^{44}$ referem como um dos problemas éticos vivenciados pelos profissionais justamente a comunicação inadequada com o paciente, com omissão de informações. Tais problemas, como dito, relacionam-se com estruturas e condições organizacionais, e as instituições deveriam estar atentas e assumir a responsabilidade pelos efeitos da comunicação sobre profissionais e pacientes. Assim, o sistema de saúde precisar criar canais de comunicação eficazes entre a equipe ${ }^{46}$, bem como desenvolver formas de diálogo franco e responsável para beneficiar a relação profissional-paciente ${ }^{37,47}$.

Outra categoria importante foi a da comunicação de incidentes, dentro da qual apareceram aspectos como medo, punição, constrangimento e pressão. O medo da punição e a exposição do nome do profissional são fatores que limitam a notificação de erros e eventos adversos ${ }^{46}$. Para reverter esse cenário é preciso garantir ao profissional uma comunicação aberta e efetiva, que o impeça de experimentar sentimentos de medo, vergonha, confusão, incerteza, insegurança e frustração ${ }^{48}$. Esses sentimentos surgem quando a concepção de ética recai toda sobre as intenções e convicções do indivíduo, fazendo-o se sentir culpado. Por outro lado, se o acento estivesse nos 
resultados, dependentes de aspectos organizacionais e tecnológicos, a dimensão ética seria compreendida na perspectiva da responsabilidade compartilhada. A comunicação do evento adverso não precisaria provocar medo e constrangimento nem estimular a tendência a esconder informações. Pelo contrário, o diálogo seria incentivado com o fim de analisar aspectos organizacionais e tecnológicos que ocasionaram o incidente, não para buscar um culpado, mas para pensar mudanças que impeçam a repetição do erro.

Os achados sobre beneficência, não maleficência, justiça e autonomia, em sua relação com as dimensões éticas da segurança, referem-se primordialmente ao aspecto tecnológico das práticas de saúde, mais do que ao aspecto organizacional. Aqui aparecem, como elementos centrais da responsabilidade, a relação não recíproca e desigual, a possibilidade de causar mal e a perspectiva de futuro das práticas de saúde. Nesse sentido, o princípio da beneficência engloba a não maleficência no compromisso de avaliar e evitar danos previsíveis. Assim aparecem as duas dimensões centrais da responsabilidade ${ }^{6}:$ a previsão e a possibilidade do mal em uma relação não recíproca e desigual, como acontece nas situações em que a segurança do paciente está implicada.

Essa mesma compreensão da responsabilidade aparece quando se entende a autonomia como capacidade de estabelecer a si mesmo as próprias leis - ou seja, capacidade de pensar, decidir e agir. Reconhecer essa capacidade, respeitando a dignidade humana do paciente e suas decisões, é um princípio ético para a conduta do profissional ${ }^{49}$. Isso significa garantir o bem-estar e os direitos do paciente, reconhecendo que, quanto mais invasivo e passível de dano for o procedimento, mais é necessário esclarecê-lo, justificá-lo e pedir consentimento ao paciente ${ }^{50}$. Uma vez que a relação clínica é desigual e não recíproca, cabe ao profissional se responsabilizar pelos resultados da ação, prevendo e evitando riscos e danos.

O tema da justiça também apareceu nas pesquisas. Um estudo ${ }^{51}$ identificou que a cultura da justiça reconhece que a desigualdade na distribuição dos recursos não depende de causas individuais, mas da ineficiência do sistema. Portanto, a responsabilidade pela injustiça e pela falha em alcançar resultados sociais prometidos e esperados é sistêmica, dependente de aspectos organizacionais.

Com relação aos elementos potencializadores da ética da segurança do paciente, a educação permanente no cotidiano profissional é apontada como caminho para criar uma cultura da segurança no ambiente de trabalho. Para Nora, Zoboli e Vieira ${ }^{44}$, esse tipo de educação pode desenvolver competências éticas. Cabe apontar, no entanto, que o modelo ideal para esse aprendizado cotidiano se basearia na ética da responsabilidade, que foca o resultado das ações, e não as convicções dos profissionais ${ }^{5}$, considerando a relação não recíproca e desigual da responsabilidade, a possibilidade de ocorrência do mal e a perspectiva de projeção para o futuro ${ }^{6}$.

Como principal resultado, recomenda-se que serviços de saúde desenvolvam diretrizes claras e baseadas em evidências para promover uma cultura organizacional voltada à segurança do paciente. Para isso, é necessário que a formação dos profissionais seja permanente e voltada sobretudo a competências éticas e à capacidade de lidar com a tecnologia. Nesse sentido, é premente desenvolver e implementar programas educacionais e de aconselhamento para profissionais, abordando princípios éticos da segurança da assistência, habilidades de comunicação, notificação de incidentes, direitos do paciente e gerenciamento de aspectos emocionais, éticos e legais.

Vale destacar que o processo de potencializar a ética na segurança do paciente não ocorre em um vácuo moral, e por isso é importante assumir o modelo da ética da responsabilidade. É preciso conhecer e entender tanto questões práticas, associadas à assistência ao paciente e aos programas de segurança, quanto questões organizacionais ${ }^{14}$. A segurança do paciente, que deve ser uma prioridade nacional ${ }^{13}$, somente será efetiva se a responsabilidade se tornar o fundamento das relações éticas nos serviços de saúde.

\section{Considerações finais}

Fatores organizacionais e da equipe, comunicação com paciente, comunicação de incidentes, beneficência e não maleficência, justiça e autonomia foram elementos identificados na presente revisão e discutidos a partir da ética 
da responsabilidade. Os resultados podem ser úteis para profissionais de saúde que atuam no cuidado aos pacientes. Uma vez que incidentes fazem parte do cotidiano dos serviços de saúde, é preciso que os profissionais estejam preparados para lidar com questões éticas a partir da perspectiva da responsabilidade. $O$ estudo pode colaborar ainda para que gestores e especialistas estejam atentos a aspectos tecnológicos e organizacionais que envolvem a segurança do paciente.

Conclui-se que é importante manter processos educativos permanentes sobre ética e segurança do paciente, com o objetivo de que profissionais desenvolvam habilidades de comunicação e sensibilidade para conduzir situações que envolvam incidentes, tomando consciência e assumindo responsabilidade pelos resultados das ações. Assim, acredita-se que quando um dano (ou possibilidade de dano) acontece durante o cuidado, esta é uma oportunidade para analisar o problema, educar a equipe sobre a cultura de segurança e criar novas pactuações em torno de práticas que promovam a responsabilidade pelos incidentes, mitigando seus efeitos.

\section{Referências}

1. Reis $\mathrm{CT}$, Martins M, Laguardia J. A segurança do paciente como dimensão da qualidade do cuidado de saúde: um olhar sobre a literatura. Ciênc Saúde Coletiva [Internet]. 2013 [acesso 24 fev 2021];18(7):2029-36. DOI: 10.1590/S1413-81232013000700018

2. Sousa P, Mendes W, editores. Segurança do paciente: conhecendo os riscos nas organizações de saúde. Rio de Janeiro: EAD/ENSP; 2014.

3. Observatorio EBE de la Fundación Index. Evidencias para unos Cuidados de Salud Seguros: conclusiones de la V Reunión sobre Enfermería Basada en la Evidencia. Evidentia [Internet]. 2009 [acesso 24 fev 2021];6(25). Disponível: https://bit.ly/3tUlaBm

4. Bonato VL. Gestão de qualidade em saúde: melhorando assistência ao cliente. O Mundo da Saúde [Internet]. 2011 [acesso 25 fev 2021];35(5):319-31. Disponível: https://bit.ly/3dV6ugZ

5. Weber M. A política como vocação. Brasília: Editora Universidade de Brasilia; 2003.

6. Jonas H. Princípio da responsabilidade: ensaio de ética para a civilização tecnológica. Rio de Janeiro: Contraponto; 2006.

7. Arksey H, O'Malley L. Scoping studies: towards a methodological framework. Int J Soc Res Methodol [Internet]. 2005 [acesso $25 \mathrm{fev}$ 2021];8(1):19-32. DOI: 10.1080/1364557032000119616

8. Levac D, Colquhoun H, O'Brien KK. Scoping studies: advancing the methodology. Implementation Sci [Internet]. 2010 [acesso 25 fev 2021];5:69. DOI: 10.1186/1748-5908-5-69

9. Tricco AC, Lillie E, Zarin W, O'Brien KK, Colquhoun H, Levac D et al. Prisma extension for Scoping Reviews (Prisma-ScR): checklist and explanation. Ann Intern Med [Internet]. 2018 [acesso 25 fev 2021];169:467-73. DOI: 10.7326/M18-0850

10. Milos P, Larraín Al. La vinculación ético-jurídica entre la gestión del cuidado y la gestión de riesgos en el contexto de la seguridad del paciente. Aquichan [Internet]. 2015 [acesso 25 fev 2021];15(1):141-53. DOI: 10.5294/aqui.2015.15.1.13

11. Pavlish C, Brown-Saltzman K, So L, Heers A, lorillo N. Avenues of action in ethically complex situations: a critical incident study. J Nurs Adm [Internet]. 2015 [acesso 25 fev 2021];45(6):311-8. DOI: 10.1097/ NNA.0000000000000206

12. Chakraborti C. Systemic negligence: why it is morally important for developing world bioethics. Dev World Bioeth [Internet]. 2015 [acesso 25 fev 2021];15(3):208-13. DOI: 10.1111/dewb.12063

13. Chadwick R. The Ethical Importance of safety. Bioethics [Internet]. 2015 [acesso 25 fev 2021]; 29(4). DOI: 10.1111/bioe.12166

14. Benedicto AS. La ética de la ignorancia (y de la incertidumbre) y la iatrogenia, un problema de salud pública. Rev Bioet Derecho [Internet]. 2019 [acesso 25 fev 2021];45:11-24. DOI: 10.1344/rbd2019.0.27782 
15. Rubio-Navarro A, Garcia-Capilla DJ, Torralba-Madrid MJ, Rutty J. Ethical, legal and professional accountability in emergency nursing practice: an ethnographic observational study. Int Emerg Nurs [Internet]. 2019 [acesso 25 fev 2021];46. DOI: 10.1016/j.ienj.2019.05.003

16. Barkhordari-Sharifabad M, Mirjalili NS. Ethical leadership, nursing error and error reporting from the nurses' perspective. Nurs Ethics [Internet]. 2019 [acesso 25 fev 2021];27(2):609-20. DOI: 10.1177/0969733019858706

17. Mangilli DC, Assunção MT, Zanini MTB, Dagostin VS, Soratto MT. Atuação ética do enfermeiro frente aos erros de medicação. Enferm Foco [Internet]. 2017 [acesso 25 fev 2021];8(1):62-6. Disponível: https://bit.ly/3tUPmgO

18. Kadivar M, Manookian A, Asghari F, Niknafs N, Okazi A, Zarvani A. Ethical and legal aspects of patient's safety: a clinical case report. J Med Ethics Hist Med [Internet]. 2017 [acesso 25 fev 2021];30(10). Disponível: https://bit.ly/32NqeN2

19. Pronovost PJ, Faden RR. Setting priorities for patient safety: ethics, accountability, and public engagement. JAMA [Internet]. 2009. [acesso 25 fev 2021];302(8):890-1. DOI: 10.1001/jama.2009.1177

20. Opel DJ, Brownstein D, Diekema DS, Wilfond BS, Pearlman RA. Integrating ethics and patient safety: the role of clinical ethics consultants in quality improvement. J Clin Ethics [Internet]. 2009 [acesso $25 \mathrm{fev}$ 2021];20(3):220-6. Disponível: https://bit.ly/3vmujE4

21. Fakih FT, Freitas GF, Secoli SR. Medicação: aspectos ético-legais no âmbito da enfermagem. Rev Bras Enferm [Internet]. 2009 [acesso 25 fev 2021];62(1):132-5. DOI: 10.1590/S0034-71672009000100020

22. Erlen JA. Patient safety, error reduction, and ethical practice. Orthopaedic Nursing [Internet]. 2007 [acesso 25 fev 2021];26(2):130-3. DOI: 10.1097/01.NOR.0000265872.57018.88

23. Johnstone MJ. Patient safety ethics and human error management in ED contexts. Australas Emerg Nurs J [Internet]. 2007 [acesso 25 fev 2021];10(1):13-20. DOI: 10.1016/j.aenj.2006.09.002

24. Lachman VD. Patient safety: the ethical imperative. Dermatol Nurs [Internet]. 2007 [acesso 25 fev 2021];16(6):401-3. Disponível: https://bit.ly/3sNFNyU

25. Arries EJ. Patient safety and quality in healthcare: nursing ethics for ethics quality. Nurs Ethics [Internet]. 2014 [acesso 25 fev 2021];21(1):3-5. DOI: 10.1177/0969733013509042

26. Kangasniemi $M$, Vaismoradi $M$, Jasper $M$, Turunen $H$. Ethical issues in patient safety: implications for nursing management. Nurs Ethics [Internet]. 2013 [acesso 25 fev 2021];20(8):904-16. DOI: $10.1177 / 0969733013484488$

27. Chiovitti RF. Theory of protective empowering for balancing patient safety and choices. Nurs Ethics [Internet]. 2011 [acesso 25 fev 2021];18(1):88-101. DOI: 10.1177/0969733010386169

28. Berner ES. Ethical and legal issues in the use of health information technology to improve patient safety. HEC Forum [Internet]. 2008 [acesso 25 fev 2021];20(3):243-58. DOI: 10.1007/s10730-008-9074-5

29. Nelson WA, Neily J, Mills P, Weeks WB. Collaboration of ethics and patient safety programs: opportunities to promote quality care. HEC Forum [Internet]. 2008 [acesso 25 fev 2021];20(1):15-27. Disponível: https://bit.ly/3xt7DDU

30. Leape LL. Ethical issues in patient safety. Thorac Surg Clin [Internet]. 2005 [acesso 25 fev 2021];15(4):493-501. DOI: $10.1016 /$ j.thorsurg.2005.06.007

31. King C. Clinical ethics: patient and provider safety. AORN J [Internet]. 2017 [acesso 25 fev 2021];106:548-51. DOI: 10.1016/j.aorn.2017.10.003

32. Sine DM, Sharpe VA. Ethics, risk, and patient-centered care: how collaboration between clinical ethicists and risk management leads to respectful patient care. J Healthcare Risk Manag [Internet]. 2011 [acesso 25 fev 2021];31(1):32-7. DOI: 10.1002/jhrm.20077

33. Clark PA. Medication errors in family practice, in hospitals and after discharge from the hospital: an ethical analysis. J Law Med Ethics [Internet]. 2004 [acesso 25 fev 2021];32(2):349-57. DOI: 10.1111/ j.1748-720x.2004.tb00481.x

34. Eaves-Leanos A, Dunn EJ. Open disclosure of adverse events: transparency and safety in health care. Surg Clin North Am [Internet]. 2012 [acesso 25 fev 2021];92(1):163-77. DOI: 10.1016/j.suc.2011.11.001 
35. Coli RCP, Anjos MF, Pereira LL. Postura dos enfermeiros de uma unidade de terapia intensiva frente ao erro: uma abordagem à luz dos referenciais bioéticos. Rev Latino-Am Enfermagem [Internet]. 2010 [acesso 25 fev 2021];18(3). DOI: 10.1590/S0104-11692010000300005

36. Giraldo P, Corbella J, Rodrigo C, Comas M, Sala M, Castells X. Análisis de las barreras y oportunidades legales-éticas de la comunicación y disculpa de errores asistenciales en España. Gac Sanit [Internet]. 2016 [acesso 25 fev 2021];30(2):117-20. DOI: 10.1016/j.gaceta.2015.11.007

37. Lavanderos S, Pedraza J, Russo Namias M, Salas SP. Dilemas éticos acerca de la revelación de errores médicos a los pacientes. Rev Méd Chile [Internet]. 2016 [acesso 25 fev 2021];144(9):1191-8. DOI: 10.4067/ S0034-98872016000900014

38. Kangasniemi M, Vaismoradi M, Jasper M, Turunen H. Ethical issues in patient safety. Nurs Ethics [Internet]. 2013 [acesso 25 fev 2021]; 20(8): 904-16. DOI: 10.1177/0969733013484488

39. Jangland E, Nyberg B, Yngman-Uhlin P. 'It's a matter of patient safety': understanding challenges in everyday clinical practice for achieving good care on the surgical ward: a qualitative study. Scand J Caring Sci [Internet]. 2017 [acesso 25 fev 2021];31(2):323-31. DOI: 10.1111/scs.12350

40. Gómez Córdoba Al, Espinosa AF. Dilemas éticos frente a la seguridad del paciente: cuidar es pensar. Aquichan [Internet]. 2006 [acesso 25 fev 2021];6(1). Disponível: https://bit.ly/3noUhUD

41. Johnstone MJ, Kanitsaki $\mathrm{O}$. The ethics and practical importance of defining, distinguishing and disclosing nursing errors: a discussion paper. Int J Nurs Stud [Internet]. 2006 [acesso 25 fev 2021];43(3):367-76. DOI: 10.1016/j.jinurstu.2005.04.010

42. Gallagher E, Alcock D, Diem E, Angus D, Medves J. Ethical dilemmas in home care case management. J Healthc Manag [Internet]. 2002 [acesso 25 fev 2021];47(2):85-96. Disponível: https://bit.ly/2PpXHtP

43. Romero MP, González RB, Calvo MSR, Fachado AA. A segurança do paciente, qualidade do atendimento e ética dos sistemas de saúde. Rev. bioét. (Impr.). [Internet]. 2018. [acesso 25 fev 2021];26(3):333-42. DOI: $10.1590 / 1983-80422018263252$

44. Nora CRD, Zoboli ELCP, Vieira M. Ethical problems experienced by nurses in primary health care: integrative literature review. Rev Gaúcha Enferm [Internet]. 2015 [acesso 25 fev 2021];36(1):112-21. Disponível: https://bit.ly/3aDBC2b

45. Biasibetti C, Hoffmann LM, Rodrigues FA, Wegner W, Rocha PK. Comunicação para a segurança do paciente em internações pediátricas. Rev Gauch Enferm [Internet]. 2019 [acesso 25 fev 2021];40. DOI: 10.1590/1983-1447.2019.20180337

46. Massoco ECP, Melleiro MM. Comunicação e segurança do paciente: percepção dos profissionais de enfermagem de um hospital de ensino. Rev Min Enferm [Internet]. 2015 [acesso 25 fev 2021];19(2):187-91. DOI: 10.5935/1415-2762.20150034

47. Edwin A. Non-disclosure of medical errors an egregious violation of ethical principles. Ghana Med J [Internet]. 2009;43(1):34-9. Disponível: https://bit.ly/3tU2S4p

48. Oliveira RM, Silva LMS, Guedes MVC, Oliveira ACS, Sánchez RG, Torres RAM. Analyzing the concept of disruptive behavior in healthcare work: an integrative review. Rev Esc Enferm [Internet]. 2016 [acesso 25 fev 2021];50(4):695-704. DOI: 10.1590/S0080-623420160000500021

49. Campos A, Oliveira DR. A relação entre o princípio da autonomia e o princípio da beneficência (e não maleficência) na bioética médica. Rev Bras Estud Polít [Internet]. 2017 [acesso 25 fev 2021];115:13-45. DOI: 10.9732/P.0034-7191.2017V115P13

50. Peruzzo Júnior L. Autonomia, cuidado e respeito: o debate sobre o prolongamento assistido da vida. Rev Bioet Derecho [Internet]. 2017 [acesso 25 fev 2021];39:121-34. Disponível: https://bit.ly/3aDtVcj

51. Sammer CE, Lykens K, Singh KP, Mains DA, Lackan NA. What is patient safety culture? A review of the literature. J Nurs Scholarsh [Internet]. 2010 [acesso $25 \mathrm{fev}$ 2021];42(2):156-65. DOI: 10.1111/ j.1547-5069.2009.01330.x

52. Wegner W, Silva SC, Kantorski KJC, Predebon CM, Sanches MO, Pedro ENR. Education for culture of patient safety: implications to professional training. Esc Anna Nery [Internet]. 2016 [acesso 25 fev 2021];20(3). DOI: 10.5935/1414-8145.20160068 
Carlise Rigon Dalla Nora - Doutora - carlise.nora@ufrgs.com.br

(D) 0000-0001-5501-2146

José Roque Junges - Doutor - roquejunges@hotmail.com

(D) 0000-0003-4675-0993

\section{Correspondência}

Carlise Rigon Dalla Nora - Rua São Manoel, 963, Rio Branco.

CEP: 90620-110. Porto Alegre/RS, Brasil.

\section{Participação dos autores}

Carlise Rigon Dalla Nora concebeu o estudo e, junto com José Roque Junges, redigiu o manuscrito, analisou os resultados, revisou criticamente o artigo e aprovou sua versão final.

Recebido: 20.1 .2020

Revisado: 13.4 .2021

Aprovado: 14.4 .2021 